This item was submitted to Loughborough's Research Repository by the author.

Items in Figshare are protected by copyright, with all rights reserved, unless otherwise indicated.

\title{
Exercise and postprandial plasma triacylglycerol concentrations in healthy adolescent boys
}

\section{PLEASE CITE THE PUBLISHED VERSION}

http://dx.doi.org/10.1249/01.mss.0000240327.31276.18

\section{PUBLISHER}

Lippincott, Williams \& Wilkins / @ The American College of Sports Medicine

\section{VERSION}

AM (Accepted Manuscript)

\section{PUBLISHER STATEMENT}

This work is made available according to the conditions of the Creative Commons Attribution-NonCommercialNoDerivatives 4.0 International (CC BY-NC-ND 4.0) licence. Full details of this licence are available at: https://creativecommons.org/licenses/by-nc-nd/4.0/

\section{LICENCE}

CC BY-NC-ND 4.0

\section{REPOSITORY RECORD}

Barrett, Laura A., John G. Morris, David J. Stensel, and Mary Nevill. 2019. "Exercise and Postprandial Plasma Triacylglycerol Concentrations in Healthy Adolescent Boys". figshare. https://hdl.handle.net/2134/17201. 


\title{
Title:
}

Exercise reduces postprandial plasma triacylglycerol concentrations in adolescent boys

\section{Authors:}

Laura A Barrett $^{1}$, John G Morris ${ }^{2}$, David J Stensel ${ }^{2}$ and Mary E Nevill ${ }^{2}$

\author{
Affiliation: \\ ${ }^{1}$ Children's Health and Exercise Research Centre, School of Sport and Health \\ Sciences, University of Exeter, United Kingdom. ${ }^{2}$ Institute of Youth Sport, \\ School of Sport and Exercise Sciences, Loughborough University, United \\ Kingdom.
}

\section{Corresponding author:}

Laura A. Barrett, Children's Health and Exercise Research Centre, School of Sport and Health Sciences, University of Exeter, Exeter, EX1 2LU, United Kingdom

Tel .: $+44(0) 1392262812$

Fax.: $+44(0) 1392264706$

E-mail: Laura.A.Barrett@exeter.ac.uk

\section{Running title:}

Exercise, triacylglycerol and adolescent boys 


\section{ABSTRACT}

PURPOSE: Atherosclerosis is initiated in childhood. Therefore reducing postprandial triacylglycerol concentrations even in young people could potentially slow atherogenic progression. This study sought to investigate if continuous exercise and intermittent games activity would reduce postprandial triacylglycerol concentrations in adolescent boys.

METHODS: Nineteen subjects were randomly assigned to either a Continuous exercise group $(N=10)$ or an Intermittent games activity group $(N=9)$ and underwent two, 2 d trials. Trials were performed a minimum of 7 days apart in a randomized order, consisting of a Rest trial and either a Continuous exercise or Intermittent games trial. In the rest trial subjects took no exercise on day 1 . On day 1 of the exercise trial subjects completed four blocks (approximately 15 min each) of uphill treadmill walking or intermittent games activity with 3 min rest between each block. On day 2, subjects came to the laboratory after an overnight fast and finger prick blood samples were obtained in the fasted state. Subjects then consumed a test meal (1.25 g fat, $1.07 \mathrm{~g}$ carbohydrate, $0.20 \mathrm{~g}$ protein, and $67 \mathrm{~kJ}$ per kg body mass). Further blood samples were collected at 30 and $45 \mathrm{~min}$ and 1, 3, 4 and $6 \mathrm{~h}$ postprandially.

RESULTS: The total area under the triacylglycerol versus time curve was lower on day 2 following exercise in both groups (Student's t-tests for correlated means, Continuous exercise group 14\%, $P=0.050$; Intermittent games group 26\%, $P=$ $0.002)$. 
CONCLUSION: Both continuous exercise and intermittent games type activity reduce postprandial triacylglycerol concentrations in adolescent boys when performed the afternoon before ingesting a high fat meal.

Key Words: Postprandial lipemia, lipid metabolism, games activity, cardiovascular disease risk 


\section{INTRODUCTION}

\section{Paragraph Number 1}

Although the clinical manifestations of coronary heart disease are not evident until adulthood, the process leading to coronary atherosclerosis is initiated during childhood and adolescence (as evident by the presence of fatty streaks and raised lesions) (12). Elevated plasma concentrations of triacylglycerol particularly in the postprandial state have been implicated in atherogenic progression (28). Therefore interventions that reduce postprandial triacylglycerol concentrations, even in young people, could potentially slow or delay this progression. Although many studies have shown that a single session of exercise can reduce postprandial triacylglycerol concentrations in adults (1, 17, 24-27), the influence of exercise on postprandial triacylglycerol concentrations in children/adolescents has yet to be examined.

\section{Paragraph Number 2}

Evidence suggests that as children and adolescents grow and mature their physiological responses to physical activity and exercise vary [for review see (5)], consequently their responses to exercise may differ from those of adults. Although it is instinctively felt that physical activity should be of benefit to children and adolescents there is relatively little research to support this view (19). Moreover, the majority of longitudinal investigations indicate that regular exercise has little influence on the lipoprotein concentrations of children and adolescents (23). However, cross sectional studies in adolescents show that those who undertake regular physical activity have higher high density lipoprotein cholesterol (HDL cholesterol) concentrations (2). While the research literature appears to be equivocal 
it is too early to discount a role of exercise in influencing cardiovascular disease risk in children and adolescents and further research is clearly warranted (4).

\section{Paragraph Number 3}

Most studies investigating the effect of exercise on blood lipid metabolism in adults have involved exercise modes such as continuous stationary cycling or treadmill walking and running. However, continuous exercise may be an inappropriate mode for children as their patterns of activity appear to be transitory, with the median duration of an activity at any level (low, medium, high) lasting only 6 s (3). If exercise is to have a beneficial effect on postprandial triacylglycerol concentrations it may be necessary to find exercise programmes involving activities, which can be easily sustained by children over a long period of time. Many children and adolescents participate in games activity such as field hockey, rugby and soccer. A Sport England survey examining trends in sport participation in young people (6 to 16 y) between 1994 - 2002 found games activity to be the most frequently undertaken activity in school physical education lessons (21). In addition soccer was found to be the most popular extra curricular sport. Hence the effects of games activity on postprandial triacylglycerol concentrations in children and adolescents would seem to warrant investigation.

\section{Paragraph Number 4}

Therefore the present study sought to investigate the effect of walking (continuous exercise) and games activity (intermittent exercise) on postprandial triacylglycerol concentrations in adolescent boys. It was hypothesized that since exercise has been 
shown to reduce postprandial triacylglycerol concentrations in adults the same relationship would be evident in adolescents.

\section{METHODS}

\section{Subjects}

\section{Paragraph Number 5}

Nineteen healthy male adolescents aged $15.4 \pm 0.1 \mathrm{yr}$ (mean \pm SE), volunteered to participate in this study, which had the approval of the university's Ethical Advisory Committee. Before any testing took place all subjects and their primary caregivers had the procedures and risks associated with involvement in the study explained to them (verbally and in writing). Written assent from each subject and consent from his primary caregiver was then obtained. All subjects were recreationally active. Subjects were randomly assigned to either the Continuous exercise group $(N=10)$ or the Intermittent games activity group $(N=9)$. The physical characteristics of the subjects are presented in Table 1. Skinfold thicknesses were measured at the following sites: triceps, biceps, subscapular and suprailiac.

\section{Preliminary tests}

\section{Paragraph Number 6}

Each subject was asked to make a self-assessment of sexual maturity using a fivepoint scale described by Tanner (22) to assess the development of genitalia and pubic hair. The scale ranges from 1 (pre-pubescent) to 5 (adult). Peak oxygen uptake (peak $\dot{\mathrm{V}} \mathrm{O}_{2}$ ) was measured directly using an incremental, uphill treadmill walking test to exhaustion. The test protocol commenced at a belt speed selected by the subject and at an initial incline of $5 \%$. The belt speed remained constant throughout the test with 
the inclination increasing by $4 \%$ at the end of every 3 min stage. The relationship between oxygen uptake, speed and gradient was also established using this test, in order to set the gradient necessary to elicit $60 \%$ of each subjects peak $\dot{\mathrm{VO}}_{2}$ at their self-selected speed for the Continuous exercise trial. Oxygen consumption and carbon dioxide production were determined using standard Douglas bag methods. In addition peak $\dot{\mathrm{VO}}_{2}$ was estimated using a progressive Multistage Shuttle Run Test (MSST) (18). The maximum speed achieved during this test was used to calculate the running speeds during the Intermittent games trial.

\section{Paragraph Number 7}

Each subject also performed a familiarization session. In both the uphill walking and the simulated games activity the target exercise intensity was $60 \%$ of peak $\dot{\mathrm{VO}}_{2}$ over each block. Subjects in the Continuous group were required to perform 4 x 15 min blocks of uphill walking with 3 min rest between each block. This replicated the amount of exercise performed during the main trials. During the walk sessions expired air collections were made during the final minute of each block using a Douglas bag attached to an adapted rucksack as this was the method used to make expired air collections during the Intermittent games activity trial. Heart rate was monitored and ratings of perceived exertion (RPE) (6) noted during each expired air collection. Duplicate capillary blood samples were taken to determine blood lactate concentration at rest and on the completion of block 1 and 4 . The gradient each subject walked at was adjusted during the familiarization session until the desired exercise intensity of $60 \%$ peak $\dot{\mathrm{VO}}_{2}$ over each block was achieved. The gradient was not adjusted during the main trial. 


\section{Paragraph Number 8}

A modified version of the Loughborough Intermittent Shuttle Test (LIST) was used to simulate games activity (15). The LIST protocol required subjects to repeat a pattern of exercise, which consisted of a walk, sprint, “cruise” and "jog” over a marked $20 \mathrm{~m}$ distance in a sports hall (see Figure 1). The average intensities of the "cruise" and “jog” phases were set at 70/40\% of estimated peak $\dot{\mathrm{V}}{ }_{2}$. During the LIST sessions an expired air collection was made during the 10th cycle of each block using a Douglas bag attached to a rucksack. Heart rate was monitored throughout the exercise sessions (Polar Electro S810, Finland) and RPE was noted just before sprint 8 of each block. Capillary blood samples were collected as in the walk sessions. Subjects in the Intermittent games group performed 2 to 4 blocks of the LIST. If the desired exercise intensity of $60 \%$ peak $\dot{\mathrm{VO}}_{2}$ over each block was not achieved using the running speeds predicted from the Multistage Shuttle Run Test, the "cruise" and “jog” speeds were lowered during the familiarization session until the intensity achieved was as close as possible to $60 \%$ of $\dot{\mathrm{V}}{ }_{2}$ max without the speeds becoming so slow subjects had to walk. Speeds were not adjusted during the main trial.

\section{Main trials}

\section{Paragraph Number 9}

Each subject took part in two main trials: a Rest trial and an Exercise trial (either Continuous or Intermittent games). The trials were separated by a minimum of $7 \mathrm{~d}$, and performed in a randomized order. Each trial was conducted over $2 \mathrm{~d}$. During the Rest trial subjects refrained from exercise on day 1. During the Exercise trials, on the afternoon of day 1 , subjects performed a 15 min warm-up on a treadmill consisting of 5 min of walking at a $1 \%$ gradient, at the speed maintained during the peak $\mathrm{VO}_{2}$ test, 
5 min of stretching and 5 min of walking at a speed and gradient selected to elicit $60 \%$ of peak $\dot{\mathrm{VO}}_{2}$. Subjects then completed either four blocks of treadmill walking or four blocks of the LIST, with 3 min rest between each block. The next morning (day 2) subjects attended the laboratory for an oral fat tolerance test. The exercise sessions were completed between 1530 and $1700 \mathrm{~h}$ hence the time interval between the end of the exercise sessions and beginning of the fat tolerance tests was approximately $16 \mathrm{~h}$. Subjects performed all exercise trials in pairs.

\section{Paragraph Number 10}

On day 2 of each trial subjects reported to the laboratory between 0900 and $0930 \mathrm{~h}$ after an overnight fast. Upon arrival in the laboratory subjects were asked to sit quietly for 10 min after which capillary blood samples were obtained. Subjects then consumed a high fat mixed meal comprising of cereal, fruit, nuts, chocolate and whipping cream, with the whipping cream being the main contributor to the fat load. The meal provided $1.25 \mathrm{~g}$ fat, $1.07 \mathrm{~g}$ carbohydrate and $0.20 \mathrm{~g}$ protein per kg body mass. A clock was started when subjects began eating. Further blood samples were collected at $0.5,0.75,1,3,4$ and $6 \mathrm{~h}$. To minimise postural variations in lipoprotein values, subjects were seated for at least 10 min before samples were taken. Subjects read, watched videos or played computer games throughout this period. Subjects consumed water ad libitum during the first trial and the volume ingested was replicated in the subsequent trial.

\section{Paragraph Number 11}

Subjects were asked to refrain from physical activity for the $2 \mathrm{~d}$ before the commencement of each trial and also to record their food and drink intake during the 
$2 \mathrm{~d}$ period before the oral fat tolerance test of their first trial. They were asked to replicate this diet for the subsequent trial.

\section{Analytical methods}

\section{Paragraph Number 12}

Capillary blood samples taken during exercise were analyzed for lactate using an enzymatic fluorimetric method (Locarte Fluorimeter Model 8-9, Locarte, London, U.K.) as described by Maughan (11). Capillary blood samples collected during the oral fat tolerance test were analyzed for total cholesterol, HDL cholesterol (fasting samples only), glucose and triacylglycerol concentration (samples taken at 0.5 and $0.75 \mathrm{~h}$ were analyzed for glucose concentration only). For glucose analysis the capillary whole blood samples were analyzed spectrophotometrically (Shimadzu Spectrophotometer, Shimadzu Corporation, Kyoto, Japan) using a commercially available kit (Randox Laboratories Ltd; UK) as described by Maughan (11). Total cholesterol and HDL cholesterol concentrations were determined using a dry chemistry method (Reflotron, Boehringer Mannheim, Germany). For triacylglycerol analysis capillary whole blood samples were collected into potassium-EDTA-coated microvette CB 300 tubes (Sarstedt Ltd, Leicester, UK) then centrifuged (EppendorfAnderman Centrifuge 5414, Germany) at $12492 \times \mathrm{g}$ for $3 \mathrm{~min}, 20 \mu \mathrm{L}$ of plasma was removed and then diluted 50 times by the addition of $980 \mu \mathrm{L}$ of ice-cold saline (155 $\mathrm{mmol} / \mathrm{L}$ ) (in order to prevent any freeze-drying effect as a result of storage) and then stored at $-20^{\circ} \mathrm{C}$ for subsequent analysis by enzymatic, colorimetric methods (Randox Laboratories Ltd, U.K.) with the use of a centrifugal analyzer (Cobas Mira Plus, Roche, Basel, Switzerland). As the samples had been pre-diluted the dilution step of the assay was omitted and 3 times the amount of sample stated in the protocol was 
used. This resulted in the concentration of the sample for analysis being the same as that in the original protocol.

\section{Statistical analysis}

\section{Paragraph Number 13}

The $6 \mathrm{~h}$ area under the plasma concentration versus time curves for triacylglycerol and glucose were calculated using the trapezium rule. The incremental area under the curve for triacylglycerol was calculated using the same method after correcting for baseline concentrations. Fasting concentrations for total cholesterol, HDL cholesterol, triacylglycerol and glucose concentrations and the area under the curve values for triacylglycerol and glucose in the two different groups were compared between trials using Student's t-tests for correlated means. Two-way ANOVA (trial time) was used to determine differences between trials and over time for postprandial plasma concentrations of triacylglycerol and glucose. Triacylglycerol data were logarithmically transformed prior to statistical analysis as these data were not normally distributed. Normality was checked via the Anderson-Darling test for normality using Minitab software release 14 for Windows (Minitab Ltd, Coventry, England). Data were analysed using the Statistical Package for Social Science (SPSS) software version 11.0 for Windows (SPSS Inc, Chicago, IL). Statistical significance was accepted at the $\mathrm{P}<0.05$ level. Results are presented as means $\pm \mathrm{SE}$.

\section{RESULTS}

\section{Paragraph Number 14}

In the following sections the results from the continuous and intermittent trials have not been directly compared. This was avoided as no attempt was made to match the 
subjects in the two groups. In addition the intensity and duration of the exercise sessions were different.

\section{Results Continuous exercise group}

\section{Reponses during exercise trial}

\section{Paragraph Number 15}

Exercise data from the Continuous trial are presented in Table 2. Averages over the four blocks of exercise are given for heart rate, \% heart rate maximum (HRmax), \% peak $\dot{\mathrm{V}} \mathrm{O}_{2}$, RPE, and block time. Blood lactate concentrations were based on an average from two measurements (post block 1 and post block 4). The mean gross energy expenditure for the Walk was $2.0 \pm 0.1 \mathrm{MJ}$ or $32.3 \pm 2.3 \mathrm{~kJ}^{\mathrm{kg}} \mathrm{kg}^{-1}$ body mass.

\section{Blood concentrations in the fasted state}

\section{Paragraph Number 16}

Blood concentrations in the fasted state are shown in Table 3. There was no difference in fasting total cholesterol, HDL cholesterol, triacylglycerol or glucose between the Rest and Continuous trials.

\section{Postprandial blood responses to the fat tolerance tests}

\section{Paragraph Number 17}

Plasma triacylglycerol responses to the test meals are shown in Figure 2 (a). The postprandial triacylglycerol concentrations were lower during the Continuous than the Rest trial. The total area under the triacylglycerol versus time curve was reduced by 14\% when the Continuous trial was compared with the Rest trial $(7.26 \pm 0.82$ versus $8.39 \pm 0.75 \mathrm{mmol} \cdot \mathrm{L}^{-1} \cdot 6 \mathrm{~h}$ respectively, main effect trial, $\left.P=0.050\right)$. The incremental 
area under the triacylglycerol versus time curve did not differ significantly between the Continuous and the Rest trial $\left(1.76 \pm 0.50\right.$ versus $1.85 \pm 0.31 \mathrm{mmol} \cdot \mathrm{L}^{-1} \cdot 6 \mathrm{~h}$ respectively).

\section{Paragraph Number 18}

The glucose responses to the test meals are shown in Figure 3 (a). There was no difference between postprandial glucose concentrations in the Rest and the Continuous trial. The total area under the concentration versus time curve for glucose did not differ between trials (Rest $27.16 \pm 0.64 \mathrm{mmol} \cdot \mathrm{L}^{-1} \cdot 6 \mathrm{~h}$ and Continuous $27.16 \pm$ $\left.0.74 \mathrm{mmol} \cdot \mathrm{L}^{-1} \cdot 6 \mathrm{~h}\right)$.

\section{Results Intermittent games group}

\section{Reponses during exercise trial}

\section{Paragraph Number 19}

Exercise data from the Intermittent games trial are presented in Table 2. Averages over the four blocks of exercise are given for heart rate, \% $\mathrm{HR}$ max, \% peak $\dot{\mathrm{VO}}_{2}$, RPE, and block time. Blood lactate concentrations were based on an average from two measurements (post block 1 and post block 4). The energy expenditure for the Intermittent games trial was not calculated due to the methodological difficulties associated with calculating energy expenditure during intermittent running involving maximal sprinting when the RER is above unity. 


\section{Blood concentrations in the fasted state}

\section{Paragraph Number 20}

Blood concentrations in the fasted state are shown in Table 3. There was no difference in fasting total cholesterol, HDL cholesterol or glucose between the Rest and Intermittent games trials, however fasting triacylglycerol concentrations were found to be lower in the Intermittent games trial $(P=0.013)$.

\section{Postprandial blood responses to the fat tolerance tests}

\section{Paragraph Number 21}

Plasma triacylglycerol responses to the test meals are shown in Figure 2 (b). The postprandial triacylglycerol concentrations were lower during the Intermittent games than the Rest trial. In addition both the total and the incremental area under the plasma triacylglycerol versus time curves were lower in the Intermittent games than the Rest trial $\left(6.92 \pm 0.79\right.$ versus $9.38 \pm 1.25 \mathrm{mmol} \cdot \mathrm{L}^{-1} \cdot 6 \mathrm{~h}$, reduced by $26 \%, P=$ $0.002 ; 1.46 \pm 0.28$ versus $2.73 \pm 0.43 \mathrm{mmol} \cdot \mathrm{L}^{-1} \cdot 6 \mathrm{~h}$, reduced by $47 \%, P=0.018$ respectively).

\section{Paragraph Number 22}

The glucose responses to the test meals are shown in Figure 3 (b). There was no difference between postprandial glucose concentrations in the Rest or the Intermittent games trial. The total area under the concentration versus time curve for glucose did not differ between trials (Rest $26.85 \pm 0.49 \mathrm{mmol} \cdot \mathrm{L}^{-1} \cdot 6 \mathrm{~h}$ and LIST $26.56 \pm 0.56$ $\left.\mathrm{mmol} \cdot \mathrm{L}^{-1} \cdot 6 \mathrm{~h}\right)$. 


\section{DISCUSSION}

\section{Paragraph Number23}

The main finding in the present study was that a single session of both continuous and intermittent games activity performed $16 \mathrm{~h}$ prior to an oral fat tolerance test reduced postprandial triacylglycerol concentrations in a group of adolescent males. The total area under the plasma triacylglycerol versus time curve was reduced by $14 \%$ ( $P=$ $0.050)$ as a result of the uphill walking and $26 \%(P=0.002)$ as a result of the games activity. As there were two separate exercise groups in this study any comparisons made between the two exercise modes are made with caution. To the knowledge of the authors this is the only study that has examined the effect of exercise on postprandial triacylglycerol concentrations in adolescents and thus the key findings of a reduction in plasma triacylglycerol as a result of walking and games activity are novel.

\section{Paragraph Number 24}

It is likely that exercise reduces postprandial triacylglycerol concentrations by the same mechanisms in adolescents as in adults, that is enhanced triacylglycerol clearance in the blood stream due to increased lipoprotein lipase (LPL) activity in the skeletal muscle (16) and/or reduced hepatic very low density lipoprotein (VLDL) secretion (8-10, 14, 20). Endogenous (VLDL) and exogenous (chylomicrons) triacylglycerol are cleared by a common saturable pathway. Consequently the lower fasting triacylglycerol concentrations seen in the Intermittent games trial when compared with the Rest trial $(P=0.013)$ could have aided increased triacylglycerol clearance. In addition, the lower incremental area (above baseline) under the triacylglycerol versus time curve in the Intermittent games trial when compared with 
the Rest trial $(P=0.018)$ may indicate decreased hepatic secretion of VLDL after exercise. However, no measure of hepatic secretion of VLDL was made in this study.

\section{Paragraph Number 25}

The $12 \%$ greater attenuation in postprandial triacylglycerol concentrations following the Intermittent games sessions in comparison with the Walk sessions may be explained by two factors. The duration of the Intermittent games sessions were on average 13 min $56 \mathrm{~s}$ longer than the Walk sessions. In addition the elevated blood lactate, heart rate and \% HR max values seen in the Intermittent games compared with the Walk sessions suggest that the exercise intensity elicited may have been higher in the Intermittent games than the Walk sessions. It is thought that energy expenditure is a key determinant of the extent to which triacylglycerol concentrations are lowered following exercise $(24,25)$. The fact that both the exercise duration and intensity were greater in the Intermittent games sessions suggests that the energy expenditure may have been higher in the Intermittent games compared with the Walk sessions.

\section{Paragraph Number 26}

The mode of exercise itself may also have influenced the results. It is important to note that the RPE elicited by the two different exercise modalities were quite close between groups. This is of interest because it suggests that for the same subjective effort games activity (the LIST) produces a greater reduction in postprandial lipemia than walking exercise. The times given to complete both the cruise and jog phases of the adapted LIST protocol used in this study were increased greatly from those in the original protocol in an attempt to reduce the overall intensity of the LIST sessions (it is important to note that the LIST was developed to simulate games activity in adults 
not adolescents so it might not be a true representation of the activity patterns seen during games activity in adolescents). However, there was a limit to how far speeds could be reduced before subjects were walking. In an actual game/match of field hockey, rugby or soccer the exercise intensity, and as a result the energy expended, may possibly be even greater. This greater energy expenditure could potentially lead to a greater reduction in postprandial triacylglycerol concentrations than that seen in this study. It should be acknowledged that the markers, which are used to indicate exercise intensity during steady state exercise, may have been artificially elevated by

the maximal sprinting element of the LIST protocol (included 40 maximal sprints). This may make them inappropriate indicators of exercise intensity during intermittent exercise involving maximal sprinting. It is also possible that the variety of different speeds that subjects were required to walk and run at and the greater level of concentration this required, may have lead to the Intermittent games sessions being more enjoyable for subjects than the walk sessions, thus resulting in lower RPE.

\section{Paragraph Number 27}

There are only a limited number of studies indicating that exercise has a beneficial influence on the lipid and lipoprotein profile of children or adolescents [for reviews see $(2,7,23)]$. Therefore the reduction in postprandial triacylglycerol concentrations seen in response to exercise in the group of adolescents in this study is perhaps unexpected. However, when differences in lipid and lipoprotein profile are detected one of the most consistent findings from cross-sectional studies in children and adolescents is that athletic or highly active children have higher levels of HDL cholesterol compared to inactive groups (19). As HDL cholesterol concentration is a marker for the efficiency of triacylglycerol metabolism in the postprandial state (13) 
the higher HDL cholesterol concentrations seen in these more active children and adolescents could be a result of exercise increasing their ability to metabolize triacylglycerol efficiently.

\section{Paragraph Number 28}

In conclusion the findings of this study indicate that both continuous exercise and intermittent games type activity can reduce postprandial triacylglycerol concentrations in adolescent boys and therefore both continuous exercise and intermittent games activity may be useful in helping to slow atherogenic progression.

\section{Acknowledgements}

The authors would like to thank the boys from Lancaster Boys College Leicestershire for participating in this study and D McCann and P Carter for their assistance with the coordination of the study.

Grants: This work was supported by a grant from the British Heart Foundation National Centre for Physical Activity and Health 


\section{REFERENCES}

1. Aldred, H. E., I. C. Perry, and A. E. Hardman. The effect of a single bout of brisk walking on postprandial lipemia in normolipidemic young adults. Metabolism. 43:836-841, 1994.

2. Armstrong, N. and B. Simons-Morton. Physical activity and blood lipids in adolescents. In: Physical activity guidelines for adolescents. J. F. Sallis (Ed.): Human Kinetics Publishers, 1994, p. 381.

3. Bailey, R. C., J. Olson, S. L. Pepper, J. Porszasz, T. J. Barstow, and D. M. Copper. The level and tempo of children's physical activities: An observational study. Med Sci Sports Exerc. 27:1033-1041, 1995.

4. Biddle, S. J. H., T. Gorely, and D. J. Stensel. Health-enhancing physical activity and sedentary behaviour in children and adolescents. J Sports Sci. 22:679-701, 2004.

5. Boisseau, N. and P. Delamarche. Metabolic and hormonal responses to exercise in children and adolescents. Sports Med. 30:405-422, 2000.

6. Borg, G. A. Perceived exertion: A note on "history" and methods. Med Sci Sports Exerc. 5:90-93, 1973.

7. Eisenmann, J. C. Blood lipids and lipoproteins in child and adolescent athletes. Sports Med. 32:297-307, 2002.

8. Fukuda, N., M. Tojho, T. Hidaka, H. Sho, and M. Sugano. Reciprocal responses to exercise in hepatic ketogenesis and lipid secretion in the rat. Ann Nutr Metab. 35:233-241, 1991.

9. Gill, J. M., G. P. Mees, K. N. Frayn, and A. E. Hardman. Moderate exercise, postprandial lipaemia and triacylglycerol clearance. Eur J Clin Invest. 31:201-207, 2001. 
10. Gill, J. M. R., K. N. Frayn, S. A. Wootton, G. J. Miller, and A. E. Hardman. Effects of prior moderate exercise on exogenous and endogenous lipid metabolism and plasma factor vii activity. Clin Sci. 100:517-527, 2001.

11. Maughan, R. J. A simple, rapid method for the determination of glucose, lactate, pyruvate, alanine, 3-hydroxybutyrate and acetoacetate on a single 20-mul blood sample. Clin Chim Acta. 122:231-240, 1982.

12. McGill, H., C., C. A. McMahan, E. Herderick, E., G. Malcom, T., R. Tracy, E., and J. Strong, P. Origin of atherosclerosis in childhood and adolescence. Am J Clin Nutr. 72(suppl):1307S-1315S, 2000.

13. Miesenböck, G. and J. R. Patsch. Postprandial hyperlipidemia: The search for the atherogenic lipoprotein. Curr Opin Lipidol. 3:196-201, 1992.

14. Mondon, C. E., C. B. Dolkas, T. Tobey, and G. M. Reaven. Causes of the triglyceride-lowering effect of exercise training in rats. $J$ Appl Physiol. 57:1466-1471, 1984.

15. Nicholas, C. W., F. E. Nuttall, and C. Williams. The loughborough intermittent shuttle test: A field test that simulates the activity pattern of soccer. J Sports Sci. 18:97-104, 2000.

16. Oscai, L. B., D. A. Essig, and W. K. Palmer. Lipase regulation of muscle triglyceride hydrolysis. J Appl Physiol. 69:1571-1577, 1990.

17. Petitt, D. S. and K. J. Cureton. Effects of prior exercise on postprandial lipemia: A quantitative review. Metabolism. 52:418-424, 2003. 
18. Ramsbottom, R., J. Brewer, and C. Williams. A progressive shuttle run test to estimate maximal oxygen uptake. Br J Sports Med. 22:141-144, 1988.

19. Rowland, T. W. The role of physical activity and fitness in children in the prevention of adult cardiovascular disease. Prog Pediatr Cardiol. 12:199-203, 2001.

20. Simonelli, C. and R. P. Eaton. Reduced triglyceride secretion: A metabolic consequence of chronic exercise. Am J Physiol. 234:E221227, 1978.

21. Sport England. Young people and sport in england. Trends in participation 1994 - 2002. London: Sport England, 2003

22. Tanner, J. M. Growth at adolescence. 2nd ed. Oxford: Blackwell Scientific Publications, 1962

23. Tolfrey, K., A. M. Jones, and I. G. Campbell. The effect of aerobic exercise training on the lipid-lipoprotein profile of children and adolescents. Sports Med. 29:99-112, 2000.

24. Tsetsonis, N. V. and A. E. Hardman. Effects of low and moderate intensity treadmill walking on postprandial lipaemia in healthy young adults. Eur J Appl Physiol. 73:419-426, 1996.

25. Tsetsonis, N. V. and A. E. Hardman. Reduction in postprandial lipemia after walking: Influence of exercise intensity. Med Sci Sports Exerc. 28:1235-1242, 1996.

26. Tsetsonis, N. V., A. E. Hardman, and S. S. Mastana. Acute effects of exercise on postprandial lipemia: A comparative study in trained and untrained middle-aged women. Am J Clin Nutr. 65:525-533, 1997. 
27. Zhang, J. Q., T. R. Thomas, and S. D. Ball. Effect of exercise timing on postprandial lipemia and hdl cholesterol subfractions. J Appl Physiol. 85:1516-1522, 1998.

28. Zilversmit, D. B. Atherogenesis: A postprandial phenomenon. Circulation. 60:473-485, 1979. 


$\begin{array}{cc}\text { Continuous } & \text { Intermittent games } \\ \text { group } & \text { group } \\ (N=10) & (N=9)\end{array}$

\begin{tabular}{|c|c|c|}
\hline Age (yr) & $15.3 \pm 0.1$ & $15.4 \pm 0.1$ \\
\hline Height (m) & $1.77 \pm 0.17$ & $1.78 \pm 0.12$ \\
\hline Body mass (kg) & $63.4 \pm 1.1$ & $59.8 \pm 1.0$ \\
\hline $\mathrm{BMI}\left(\mathrm{kg} \cdot \mathrm{m}^{-2}\right)$ & $20.3 \pm 0.5$ & $19.0 \pm 0.3$ \\
\hline Sum of 4 skinfolds (mm) & $40.9 \pm 7.3$ & $40.9 \pm 1.4$ \\
\hline Waist/hip ratio & $0.77 \pm 0.01$ & $0.77 \pm 0.01$ \\
\hline Systolic blood pressure (mmHg) & $105 \pm 2$ & $112 \pm 4$ \\
\hline Diastolic blood pressure (mmHg) & $69 \pm 4$ & $64 \pm 3$ \\
\hline Tanner stage genital - median (range) & $4(3-5)$ & $4(4-5)$ \\
\hline Tanner stage pubic hair - median (range) & $5(3-5)$ & $5(4-5)$ \\
\hline Peak $\dot{\text { VO}} 2\left(\mathrm{~mL} \cdot \mathrm{kg}^{-1} \cdot \mathrm{min}^{-1}\right)$ - treadmill & $44.8 \pm 3.4$ & $51.1 \pm 2.5$ \\
\hline Peak $\dot{\mathrm{VO}} 2\left(\mathrm{~mL} \cdot \mathrm{kg}^{-1} \cdot \mathrm{min}^{-1}\right)$ - predicted (MSST) & $48.0 \pm 3.6$ & $47.9 \pm 1.2$ \\
\hline
\end{tabular}

Values are means \pm SE. MSST, Multistage Shuttle Run Test. 
TABLE 2. Blood lactate, heart rate, percentage of maximum heart rate (\% HR max), percentage of peak oxygen uptake (\% peak $\dot{\mathrm{V}} \mathrm{O}_{2}$ ), rate of perceived exertion (RPE) and time to complete each exercise block (Block time) during the Continuous and Intermittent games trials

\begin{tabular}{lcc}
\hline & $\begin{array}{c}\text { Continuous trial } \\
(N=10)\end{array}$ & $\begin{array}{c}\text { Intermittent trial } \\
(N=9)\end{array}$ \\
\hline Blood lactate $\left(\mathrm{mmol} \cdot \mathrm{L}^{-1}\right)$ & $2.0 \pm 0.3$ & $2.4 \pm 0.7$ \\
Heart rate (beats $\left.\cdot \mathrm{min}^{-1}\right)$ & $143 \pm 3$ & $161 \pm 4$ \\
\% HR max & $73.7 \pm 1.4$ & $80.3 \pm 1.7$ \\
\% Peak VंO treadmill & $58.9 \pm 7.1$ & $68.8 \pm 2.3$ \\
RPE median (range) & $11(9-13)$ & $12(6-18)$ \\
Block time (min:s) & $15: 00 \pm 00: 00$ & $18: 29 \pm 00: 08$ \\
\hline
\end{tabular}

Values are means $\pm \mathrm{SE}$. 
TABLE 3. Fasting concentrations of blood total cholesterol, plasma high density lipoprotein (HDL) cholesterol, plasma triacylglycerol and blood glucose in the Rest and Exercise trials for the Continuous and Intermittent Games groups

\begin{tabular}{lcccc}
\hline & \multicolumn{2}{c}{ Continuous group } & \multicolumn{2}{c}{ Intermittent games group } \\
& Rest & Exercise & Rest & Exercise \\
& & & & \\
& & & \\
& & & \\
& & & \\
Blood total cholesterol $\left(\mathrm{mmol} \cdot \mathrm{L}^{-1}\right)$ & $3.50 \pm 0.10$ & $3.50 \pm 0.17$ & $3.19 \pm 0.06$ & $3.16 \pm 0.08$ \\
Plasma HDL cholesterol $\left(\mathrm{mmol} \cdot \mathrm{L}^{-1}\right)$ & $0.89 \pm 0.07$ & $0.90 \pm 0.05$ & $0.91 \pm 0.10$ & $0.89 \pm 0.09$ \\
Plasma triacylglycerol $\left(\mathrm{mmol} \cdot \mathrm{L}^{-1}\right)$ & $1.09 \pm 0.12$ & $0.93 \pm 0.08$ & $1.11 \pm 0.15$ & $0.92 \pm 0.12 *$ \\
Blood glucose $\left(\mathrm{mmol} \cdot \mathrm{L}^{-1}\right)$ & $4.46 \pm 0.14$ & $4.27 \pm 0.15$ & $4.46 \pm 0.09$ & $4.29 \pm 0.14$
\end{tabular}

Values are means \pm SE; Means (Rest and Exercise) were compared using the

Student's t-tests for correlated data. ${ }^{*}$ different from Rest, $P=0.013$. 


\section{Legends for figures}

FIGURE 1: The Loughborough Intermittent Shuttle Test (LIST) protocol. Subjects were allowed 13 s to complete each of the three $20 \mathrm{~m}$ walks, the sprint was maximal, and the intensity of the cruise and jog phases of the test was set relative to each subjects peak $\dot{\mathrm{VO}}_{2}$ as estimated on the Multistage Shuttle Run Test. The average intensity of the cruise and jog phases in the study was set at 70/40\% of estimated peak $\dot{\mathrm{V}} \mathrm{O}_{2}$. This pattern was repeated 10 times forming one "block" of exercise.

FIGURE 2: (a) Fasting and postprandial triacylglycerol concentrations for Rest and Continuous trials. Values are means $\pm \mathrm{SE} ; N=10$, black rectangle indicates consumption of test meals. Data were analysed using two-way ANOVA with repeated measures. Main effect trial, $P=0.049$; main effect time, $P<0.001$; interaction time - trial, NS.

(b) Fasting and postprandial triacylglycerol concentrations for Rest and Intermittent games trials. Values are means $\pm \mathrm{SE} ; N=9$, black rectangle indicates consumption of test meals. Data were analysed using two-way ANOVA with repeated measures. Main effect trial, $P=0.005$; main effect time, $P<0.001$; interaction trial - time, NS.

FIGURE 3: (a) Fasting and postprandial glucose concentrations for Rest and Continuous trials. Values are means $\pm \mathrm{SE} ; N=10$, black rectangle indicates consumption of test meals. Data were analysed using two-way ANOVA with repeated measures. Main effect trial, NS; main effect time, $P<0.001$; interaction trial - time, NS.

(b) Fasting and postprandial glucose concentrations for Rest and Intermittent games trials. Values are means $\pm \mathrm{SE} ; N=9$, black rectangle indicates consumption of test 
meals. Data were analysed using two-way ANOVA with repeated measures. Main effect trial, NS; main effect time, $P<0.001$; interaction trial - time, NS. 
FIGURE 1

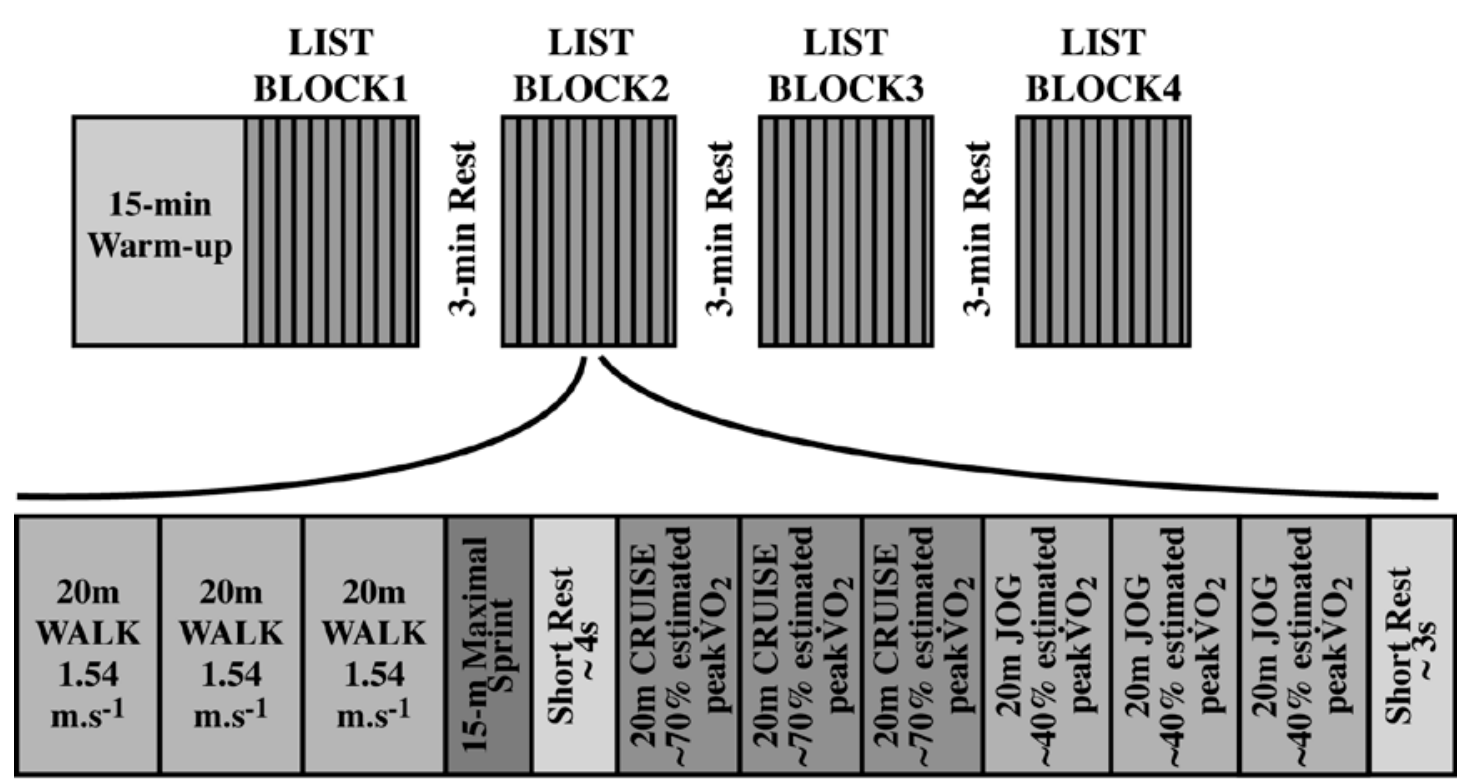

Pattern repeated 10 times to form one 'BLOCK' of exercise 
FIGURE 2

(a) Continuous exercise trial

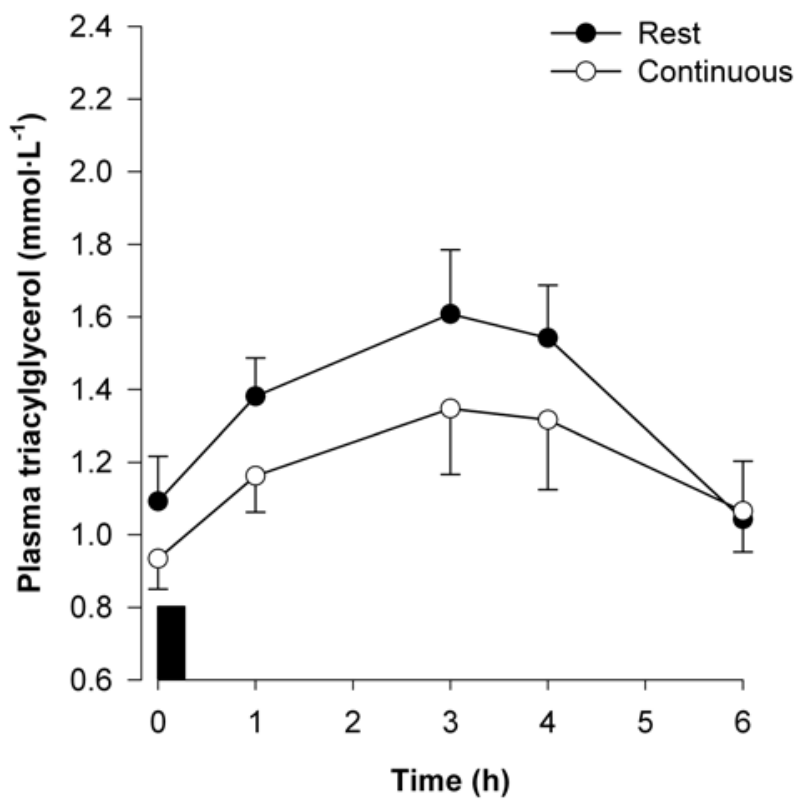

(b) Intermittent games trial

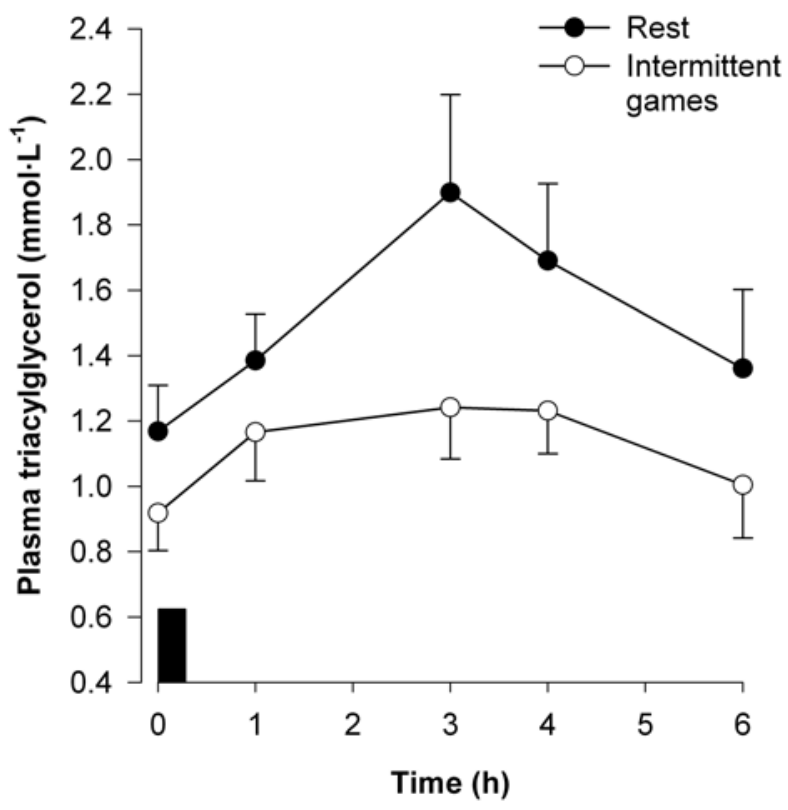


FIGURE 3

(a) Continuous exercise trial

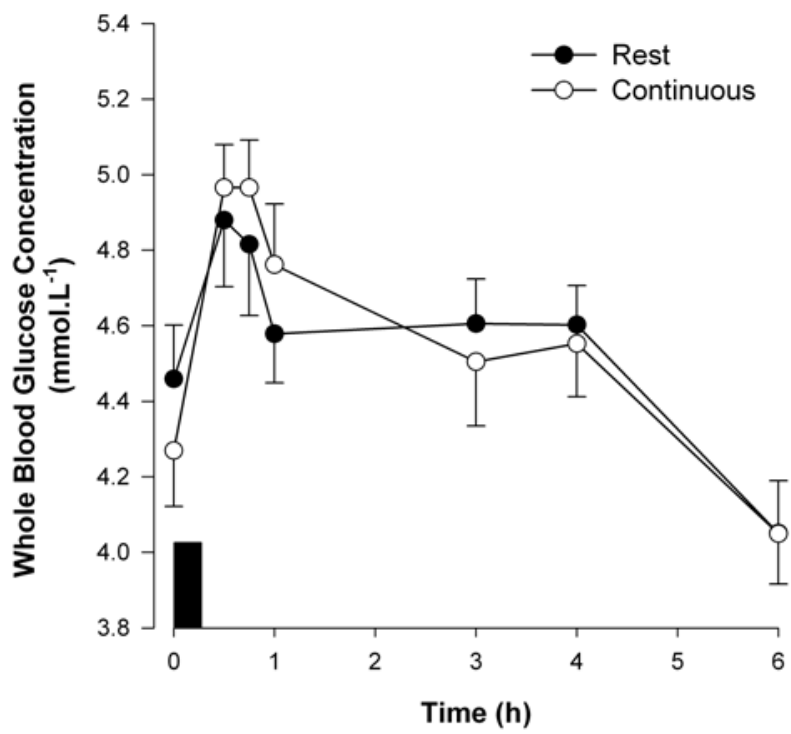

(b) Intermittent games trial

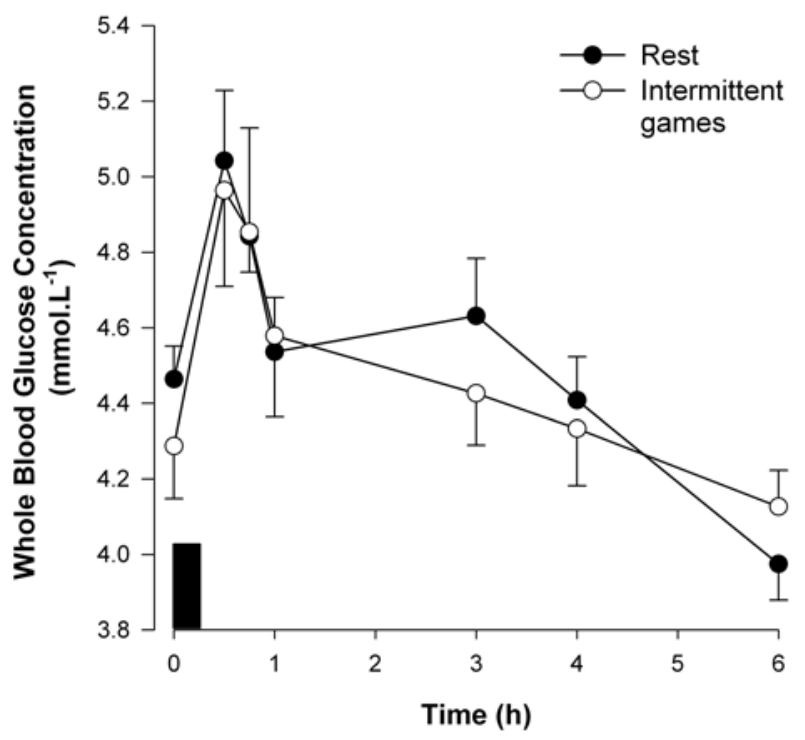

